\title{
ATMOSPHERES OF PLANETS AND THEIR SATELLITES
}

\author{
DARRELL F. STROBEL \\ Departments of Earth and Planetary Sciences \& \\ Physics and Astronomy \\ The Johns Hopkins University \\ Baltimore, MD 21218 USA
}

\begin{abstract}
A general review of the chemistry of planetary atmospheres was given with the emphasis on the outer planets and their satellites. The impact of the scientific findings of the Voyager Mission on our understanding of the chemistry and composition of the Jovian, Saturnian, and Uranian atmospheres was highlighted. Hydrocarbon photochemistry will be treated in the context of comparative planetary atmospheres and illustrated by important similarities and differences. The interesting chemistry of satellites with significant atmospheres, Titan, Triton, and Io was explored in the evolutionary context and the impact on the magnetospheres of their parent planet.

This subject matter is available in written form and may be found in the following references:

Strobel, D. F. (1983) 'Photochemistry of the Reducing Atmospheres of Jupiter, Saturn, and Titan', Intl. Rev. Phys. Rev. 3, 145.

Strobel, D. F. (1985) 'The Photochemistry of the Atmospheres of the Outer Planets and Their Satellites', in J. S. Levine (ed.), The Photochemistry of Atmospheres, Academic Press, New York, pp. $393-434$.
\end{abstract}

